\title{
Applications to Mental Health Review Boards by institutions in Gauteng
}

To the Editor: In a recent issue of SAJP', Moosa and Jeenah concluded from their review of the 2008 applications for involuntary admission to Gauteng Mental Health Review Boards (MHRBs) that it would appear that in the 4 years since promulgation of the Mental Health Care Act (MHCA) there have been significant strides towards implementation of the procedures relating to involuntary admission and care of mental health care users by all stakeholders.

Helen Joseph Hospital $(\mathrm{HJH})$ in Auckland Park, Johannesburg, in southern Gauteng province, is a 480-bed regional hospital in an urban setting. The psychiatric unit of the hospital is designated as a 72-hour assessment unit. It is a 30-bed acute adult (male and female) unit providing inpatient, outpatient and consultation/ liaison service delivery, as well as teaching and research. After initial assessment and treatment, service users are transferred to other longer-term and specialised units (e.g. adolescent, psychotherapy and eating disorder units) of the $H$ Moross Centre (Tara) and Sterkfontein Hospital (SFH), the two psychiatric hospitals on the local circuit. Alternatively, users are discharged for follow-up at the $\mathrm{HJH}$ outpatient clinic and the community psychiatric services in southern Gauteng, or for longer-term placement at a Life Health Esidimeni facility or non-governmental organisation. Three health districts exist in the southern Gauteng area Uohannesburg Metro, Ekurhuleni - East Rand, West Rand), with two MHRBs for the region (MHRB for Region A - Joburg Metro and West Rand; and Region B - Ekurhuleni).

It has been observed in previous studies at this unit, that a number of users were re-admitted to the $\mathrm{HJH}$ psychiatric unit in a 'revolving door' pattern, while some others also remained for an extended stay awaiting placement in a long-term facility. ${ }^{2,3}$ From these studies it also became clear that in order to gain a full perspective on the outcome of psychiatric services rendered at $\mathrm{HJH}$, it would be necessary to track the progress of inpatient users after discharge. In a subsequent follow-up study, a cohort of patients admitted to the acute psychiatric unit at $\mathrm{HJH}$ during 2007 was tracked in order to assess their progress and establish their legal status after 12 and 24 months.

The routine administrative data of these users were reviewed on admission to and discharge from $\mathrm{HJH}$, as well as to and from the facilities/services to which they were subsequently transferred (e.g. SFH and Tara). The data used consisted of the required completed forms, including MHCA forms 1, 4, 5, 7 and 6 for admissions and MHCA forms 8, 11 and 3 on discharge. These data were compared with information that the relevant MHRBs (Regions A and B) keep on the referral of these users. The variables used to assess user progress included specification of the section of MHCA on admission, and of the section of MHCA on discharge.

In this study it was found that the MHRB data for regions $A$ and $B$ were not available in a format that made it possible to correlate and track the transfer and changing legal status of users in the system from one facility to the other. Data from the MHRB database represented record entries and not users; in other words, several unrelated records may exist for the same user, or no record may exist for others. No overview of the total patients in any facility at a specific time (e.g. monthly) was routinely obtained by the MHRBs, with the result that no conclusions could be drawn about the completeness of their records. Although there has also apparently been under-reporting of admissions to $\mathrm{HJH}$ during 2007, and forms might have been lost, there also seems to have been no follow-up action regarding this matter by the MHRBs.

It should therefore be concluded that the quality of the referral procedures and administrative record keeping of the MHRBs must be dramatically improved. An effective and relevant tracking system, without which human rights of mental health care users will continue to be compromised, must be ensured.

\section{Bernard Janse van Rensburg}

Helen Joseph Hospital

PO Box 1247

Pinegowrie

2123

. Moosa MYH, Jeenah FY. A review of the applications for involuntary admissions made to the Mental Health Review Boards by institutions in Gauteng in 2008. South African Journal of Psychiatry 2010;16(4):125-130.

2. Janse van Rensburg ABR. Clinical profile of acutely ill psychiatric patients admitted to a general hospital psychiatric unit. African Journal of Psychiatry 2007; 10(3):59-163.

3. Janse van Rensburg ABR. Acute mental health care and South African mental health legislation. Part I-Morbidity, treatment and outcome. African Journal of Psychiatry 2010; 13(5):382-389.

4. Janse van Rensburg ABR. Tracking the legal status of a cohort of inpatients on discharge from a 72 -hour assessment unit. Presented at the 16 th National Congress of the South African Society of Psychiatrists, 16 - 20 October 2010, East London. 